\title{
Bell's Palsy Associated with SARS-CoV-2 Infection in a 2-Year-Old Child
}

\author{
Serina Bsales ${ }^{10}$ Birk Olson ${ }^{1}$ Sunanda Gaur ${ }^{2,3,4}$ \\ Priyanka Uprety ${ }^{6}$ Abdolreza Esfahanizadeh ${ }^{7}$ \\ ${ }^{1}$ Department of Medicine, Rutgers Robert Wood Johnson Medical \\ School, New Brunswick, New Jersey, United States \\ 2 Department of Pediatrics, Division of Allergy, Immunology and \\ Infectious Diseases, Rutgers Robert Wood Johnson Medical School, \\ New Brunswick, New Jersey, United States \\ ${ }^{3}$ Department of Epidemiology, Rutgers School of Public Health, New \\ Brunswick, New Jersey, United States \\ ${ }^{4}$ Ernest Mario School of Pharmacy, Rutgers, The State University of NJ, \\ New Brunswick, New Jersey, United States \\ ${ }^{5}$ Division of Pediatric Hospital Medicine, Rutgers Robert Wood \\ Johnson Medical School, New Brunswick, New Jersey, United States \\ 6 Department of Pathology and Laboratory Medicine, Rutgers Robert Wood \\ Johnson Medical School, New Brunswick, New Jersey, United States \\ 7 Department of Pediatrics, Division of Child Neurology and \\ Neurodevelopmental Disability, Child Health Institute of New Jersey \\ and Rutgers Robert Wood Johnson Medical School, New Brunswick, \\ New Jersey, United States \\ J Pediatr Neurol 2021;19:440-442.
}

Dalya Chefitz $^{5}$ Mary Carayannopoulos 6

\author{
Address for correspondence Serina Bsales, BS, Department of \\ Medicine, Rutgers Robert Wood Johnson Medical School, 675 Hoes \\ Lane West, Piscataway, NJ 08854, United States \\ (e-mail: srb237@rwjms.rutgers.edu).
}

\begin{abstract}
Keywords

- Bell's palsy

- COVID-19

- SARS-CoV-2

- facial nerve palsy

- facial asymmetry

Bell's palsy (BP) is an acute, unilateral facial nerve palsy (FNP) that is a diagnosis of exclusion, sometimes associated with infectious causes. In this article, we described a previously healthy 2 -year-old child patient who presented with left-sided facial droop, positive severe acute respiratory syndrome-coronavirus-2 (SARS-CoV-2) real-time reverse transcription polymerase chain reaction (RT-PCR), positive SARS-CoV-2 immunoglobulin (Ig)-G antibody, and negative cerebrospinal fluid (CSF) SARS-CoV-2 (PCR and serology). This is the second reported pediatric case of BP in the setting of SARSCoV-2, and the first in a child without comorbidities. Due to the positive antibody test, we presented the idea that SARS-CoV-2 could be a triggering factor of the FNP, possibly occurring in the later stages of disease.
\end{abstract}

\section{Introduction}

Bell's palsy (BP) is the broadly used term for idiopathic facial nerve palsy (FNP). In children, idiopathic BP is the most common cause of FNP, ${ }^{1}$ though several infectious causes have been identified. ${ }^{2} \mathrm{BP}$ onset is typically sudden, unilateral, and appreciable upon cranial nerve testing. Treatment includes steroids, antivirals, and antibiotics as appropriate, and prognosis is generally good when addressed promptly. ${ }^{3}$

received

November 5, 2020

accepted after revision

November 21, 2020

published online

January 27, 2021

As the severe acute respiratory syndrome-coronavirus-2 (SARS-CoV-2) pandemic continues, a growing diversity of peripheral nervous system manifestations has been reported, notably, disturbances in taste and smell and case reports of Guillain-Barre syndrome (GBS) and BP. ${ }^{4} \mathrm{BP}$ with confirmed SARS-CoV-2 has been reported in three adults ${ }^{5-7}$ and one child with comorbidities. ${ }^{8}$ We are reporting a case of an otherwise healthy child with BP in the setting of SARSCoV-2 infection.

(c) 2021. Thieme. All rights reserved. Georg Thieme Verlag KG,

Rüdigerstraße 14,

70469 Stuttgart, Germany
DOI https://doi.org/ 10.1055/s-0040-1722210. ISSN 1304-2580. 


\section{Case Report}

A 2-year-old male child patient with no significant past medical history presented to our emergency department in June 2020 with a left-sided facial asymmetry, most noticeable while crying, which began 1 day prior. He had an unwitnessed fall from assumed standing height; his caregiver was unsure if he hit his head, but he did not complain of pain. No reported loss of consciousness, emesis, altered behavior, or injuries. He was evaluated at an emergency department and discharged. A few hours later, his caregiver noticed the boy's left eye was not fully closing and his mouth drooped while crying. She brought him to his Primary Medical Doctor (PMD), who noted left-sided facial palsy and sent him to our emergency department.

The patient did not have recent fever, tick bite, rash, sick contacts, or exposure to novel coronavirus disease 2019 (COVID-19). There was no history of eye discharge, congestion, cough, chest pain, urinary complaints, joint swelling, or emotional stress. No known medical conditions or allergies, and he was not on medication. Family history was noncontributory. He lived at home with his caregiver and three other adults. His developmental milestones were normal. On examination, he was afebrile, weight was at the 29th percentile, and head circumference was at the 30th percentile. His vitals were as follows: 156 beats/minute; respiratory rate, 22/min; blood pressure, 95/64 mm $\mathrm{Hg}$; and $\mathrm{SpO}_{2}, 98 \%$ on room air. He was crying but consolable and not lethargic. His skin had no rashes, lesions, appreciable swelling, and no tenderness or bony defect on palpation of skull. There was no conjunctival pallor, hemotympanum, epistaxis, tonsillar erythema or exudate, stridor, lymphadenopathy, or meningismus. Lung sounds were clear and equal bilaterally; cardiac rate and rhythm were normal without murmurs or gallops. He was unable to close his left eye or wrinkle left side of his forehead while crying, and there was drooping of left angle of his mouth and he was measured grade IV on the House-Brackmann facial paralysis scale. Cranial nerves $(\mathrm{CN}) 2$ to 4,6 , and 8 to 12 were intact without deficits; he had normal bulk and tone, intact reflexes $(2+)$, moved all extremities, and did not have a tremor or abnormal movements. A noncontrast head computed tomography (CT) ruled out intracranial hemorrhage or fracture. The patient was admitted for further management.

Due to referring physician's clinical suspicion, the patient was tested for COVID-19 and found to be SARS-CoV-2 positive by real-time reverse transcription polymerase chain reaction (RT-PCR) analysis performed on a nasopharyngeal (NP) swab sample. Laboratory results were significant for white blood cell (WBC) count of 13.6 thousand/ $\mu \mathrm{L}$, with a differential of neutrophils, 53.8\%; lymphocytes, $38.0 \%$; monocytes, 6.2\%; eosinophils, $1.5 \%$; and basophils, $0.6 \%$. Cerebrospinal fluid (CSF) was colorless; total nucleated cells, $3 / \mathrm{mm}^{3}$; monocytes, $1 / \mathrm{mm}^{3}$; and lymphocytes, $2 / \mathrm{mm}^{3}$; glucose was $59 \mathrm{mg} / \mathrm{dL}$ and protein was $15 \mathrm{mg} / \mathrm{dL}$. Further CSF studies were negative for infectious etiologies: Lyme (PCR), bacterial culture, BioFire FilmArray Meningitis/Encephalitis panel (multiplex PCR for 14 pathogens), and SARS-CoV-2 (PCR and serology). The SARS-CoV-2 RT-PCR assay on Ce- pheid platform is not Food and Drug Administration (FDA) authorized for use in CSF specimens. This specimen type has not been validated in our laboratory and this assay was for research use only. Similarly, Abbott Architect, the antibody detection platform used for CSF, was not FDA approved for use in CSF; it was for research use only. He had a slightly elevated partial thromboplastin time (PTT) of 36 seconds and slightly elevated lactate dehydrogenase (LDH) of $257 \mathrm{IU} / \mathrm{Lz}$. D-Dimer ( $<215 \mathrm{ng} / \mathrm{mL})$, ferritin (45 ng/mL), fibrinogen (242 $\mathrm{mg} / \mathrm{dL})$, ProBNP $(240 \mathrm{pg} / \mathrm{mL})$, and troponin T $(<0.01 \mathrm{ng} / \mathrm{mL})$ were within normal limits. Herpes simplex virus (HSV) type1 immunoglobulin (Ig)-G antibody was reactive, cytomegalovirus IgG antibody was positive, HSV type-2 IgG antibody was nonreactive, cytomegalovirus IgM was negative. Blood Epstein-Barr virus (EBV) by PCR was undetected, herpes virus 6 DNA was $<500$ copies/mL, herpes virus 7 DNA was $<500$ copies/mL, and Lyme IgG and IgM antibodies were nonreactive. Subsequently, SARS-CoV-2 IgG antibody was reported positive. The patient was started on prednisolone $1 \mathrm{mg} / \mathrm{kg} /$ day during his second hospital day.

His left-sided facial palsy did not improve throughout his admission, and he did not develop any fever, cough, respiratory, cardiac, abdominal symptoms, or new neurological issues suggestive of SARS-CoV-2 infection. He was discharged on hospital day 3 on a prednisolone taper (1 $\mathrm{mg} / \mathrm{kg} /$ day for 3 days, then $0.75 \mathrm{mg} / \mathrm{kg} /$ day for 2 days, then $0.25 \mathrm{mg} / \mathrm{kg} /$ day for 1 day). According to the patient's caregiver, his facial weakness resolved to a House-Brackmann grade II within a week of discharge, and as per his PMD, the facial palsy was completely resolved by 4 weeks, HouseBrackmann grade I.

\section{Discussion}

We presented a case of BP in a 2-year-old, previously healthy child who was simultaneously infected with SARS-CoV-2. An association between SARS-CoV-2 and BP may be emerging as three adults and one other child have been reported to date: (1) a symptomatic (fever/myalgias) 48-year-old diabetic female with ground-glass opacities on chest CT, (2) a symptomatic (myalgias) 36-year-old male, (3) an asymptomatic 65-year-old female, and (4) an asymptomatic 6-year-old boy with significant comorbidities (chromosome abnormalities, congenital defects, asthma, obstructive sleep apnea, and agammaglobulinemia with hyper Ig M $){ }^{5-8} \mathrm{BP}$ in the context of positive SARSCoV-2 PCR has presented with signs of peripheral FNP that improved with antiviral, antibiotic, and/or steroid treatment. Respiratory symptoms were mostly absent, albeit two cases presented with myalgia and one had associated fever that could be attributed to SARS-CoV-2 infection. Traumatic and other viral causes were not identified in any case. With the exception of diabetes mellitus in the 48-year-old female, all cases lacked known risk factors for BP.

Similar to the reported cases, our patient presented without respiratory symptoms or active non-SARS-CoV-2 viral infection. Our patient's facial palsy presented hours after an unwitnessed fall; however, trauma was excluded by physical examination and head CT. Physical examination of 
our patient and previous cases are suggestive of a peripheral lesion. CSF negative for SARS-CoV-2 PCR provides additional support for a peripheral lesion not available in previous cases. Conversely, SARS-CoV-2 was detected in the CSF of patients complicated by encephalitis and encephalomyelitis, thus demonstrating BBB penetration by SARS-CoV-2.9,10

Our patient was RT-PCR and antibody positive, suggesting that he was likely infected at least 7 days prior. ${ }^{11}$ Studies have shown that viral positivity following initial infection can last up to 3 months. ${ }^{12}$ The other reported cases of BP were positive for SARS-CoV-2 by PCR, but antibody testing was not reported. Our case provides additional support for the emerging relationship between BP and SARS-CoV-2. While HSV-1 IgG was also reactive, seroprevalence in pediatric populations is high (31\%), ${ }^{13}$ hence we do not believe this child's BP was causally related to HSV-1. Postinfectious phenomena, such as multisystem inflammatory syndrome in children (MIS-C), have been a focal point of SARS-CoV-2 investigation in children. For poorly understood reasons, children generally present with milder symptoms but can also have severe atypical postinfectious presentations.

Other clinical entities involving peripheral nerve disease have been observed in the context of SARS-CoV-2 infection, specifically GBS. The proposed immune-mediated mechanisms of GBS and BP share many features, ${ }^{14}$ thus the appearance of both in the context of SARS-CoV-2 warrants investigation. Twelve cases of GBS have been reported, and similar to our patient, CSF samples were negative for SARSCoV-2. ${ }^{15}$ In the 11 nonvariant cases, SARS-CoV-2 symptoms preceded GBS onset by at least 5 days. The presence of SARS$\mathrm{CoV}-2$ antibodies in our patient may suggest a similar timeline to nerve dysfunction.

It must be acknowledged that SARS-CoV-2 and BP may not be causally related. The annual incidence of $\mathrm{BP}$ ranges from 15 to 20 per 100,000 cases. ${ }^{16}$ Given the distressing nature of facial asymmetry and its possible causes (e.g., stroke), few cases go unexamined. Meanwhile, the SARS-CoV-2 pandemic has infected over 45 million worldwide. It possible that this patient's presentation was a coincidence, this patient presented as the incidence of SARS-CoV-2 was declining ${ }^{17}$ in the region, and the incidence of FNP has increased during the SARS-CoV-2 pandemic, ${ }^{18}$ which suggests a relationship between his BP and SARS-CoV-2 diagnosis.

\section{Conclusion}

As the pandemic continues, new associations and manifestations of COVID-19 in children are being reported expanding our understanding of the full breadth of the disease in children. Here we described a case of BP in association with SARS-CoV-2 infection in a previously healthy child and propose that this may have been a postinfectious complication. Larger studies of SARS-CoV-2 PCR and antibody testing in BP are needed to better understand this association.

\section{Funding}

Research reported in this publication was supported (in part) by the New Jersey Alliance for Clinical and Transla- tional Science and the National Center for Advancing Translational Sciences (NCATS), a component of the National Institute of Health $(\mathrm{NIH})$ under award number UL1TR003017. The content is the sole responsibility of the authors and does not necessarily represent the official views of the National Institutes of Health.

\section{Conflict of Interest}

None declared.

\section{References}

1 Psillas G, Antoniades E, Ieridou F, Constantinidis J. Facial nerve palsy in children: a retrospective study of 124 cases. J Paediatr Child Health 2019;55(03):299-304

2 Cook SP, Macartney KK, Rose CD, Hunt PG, Eppes SC, Reilly JS. Lyme disease and seventh nerve paralysis in children. Am J Otolaryngol 1997;18(05):320-323

3 Karatoprak E, Yilmaz S. Prognostic factors associated with recovery in children with Bell's palsy. J Child Neurol 2019;34(14): 891-896

4 Nepal G, Rehrig JH, Shrestha GS, et al. Neurological manifestations of COVID-19: a systematic review. Crit Care 2020;24(01):421

5 Elkhouly A, Kaplan AC. Noteworthy neurological manifestations associated with COVID-19 infection. Cureus 2020;12(07):e8992

6 Mehta S, Mackinnon D, Gupta S. Severe acute respiratory syndrome coronavirus 2 as an atypical cause of Bell's palsy in a patient experiencing homelessness. CJEM 2020; 2:1-3

7 Wan Y, Cao S, Fang Q, Wang M, Huang Y. Coronavirus disease 2019 complicated with Bell's palsy: a case report. Research Square 2020 (e-pub ahead of print). Doi: 10.21203/rs.3.rs-23216/v1

8 Theophanous C, Santoro J, Itani R. Bell's palsy in a pediatric patient with hyper IgM syndrome and severe acute respiratory syndrome coronavirus 2 (SARS-CoV-2). Brain Dev 2021;43(2): 357-359

9 Moriguchi T, Harii N, Goto J, et al. A first case of meningitis/ encephalitis associated with SARS-coronavirus-2. Int J Infect Dis 2020;94:55-58

10 Bernard-Valnet R, Pizzarotti B, Anichini A, et al. Two patients with acute meningoencephalitis concomitant with SARS-CoV-2 infection. Eur J Neurol 2020;27(09):e43-e44

11 Lei Q Li Y, Hou HY, et al. Antibody dynamics to SARS-CoV-2 in asymptomatic COVID-19 infections. Allergy 2021;76(02):551-561

12 Findings from Investigation and Analysis of re-positive cases. Available at: https://www.cdc.go.kr/board/board.es?mid= a30402000000\&bid $=0030 \&$ act $=$ view\&list_no $=367267 \&$ nPage $=$ 1 external. Accessed October 5, 2020

$13 \mathrm{Xu}$ F, Lee FK, Morrow RA, et al. Seroprevalence of herpes simplex virus type 1 in children in the United States. J Pediatr 2007;151 (04):374-377

14 Greco A, Gallo A, Fusconi M, Marinelli C, Macri GF, de Vincentiis M. Bell's palsy and autoimmunity. Autoimmun Rev 2012;12(02): 323-328

15 Carrillo-Larco RM, Altez-Fernandez C, Ravaglia S, Vizcarra JA. COVID-19 and Guillain-Barre syndrome: a systematic review of case reports. Wellcome Open Res 2020;5:107-107

16 Warner MJ, Hutchison J, Varacallo M. Bell palsy. In: StatPearls. Treasure Island, FL: StatPearls Publishing; 2020

17 Arco M. NJ coronavirus death toll increases to 12,214 with 164,497 total cases. Stage 2 of reopening 1 week away. Available at: https://www.nj.com/coronavirus/2020/06/nj-coronavirusdeath-toll-increases-to-12214-with-164497-total-cases-stage2-of-reopening-1-week-away.html. Accessed November 11, 2020

18 Zammit M, Markey A, Webb C. A rise in facial nerve palsies during the coronavirus disease 2019 pandemic. J Laryngol Otol 2020; $1-4: 1-4$ 